\title{
TERCIARIZACIÓN ECONÓMICA Y PRODUCTIVIDAD AGREGADA: UN ANÁLISIS PARA ECONOMÍAS DESARROLLADAS Y EN DESARROLLO
}

TERTIARIZATION AND AGGREGATE PRODUCTIVITY: AN ANALYSIS FOR DEVELOPED AND DEVELOPING ECONOMIES

Recibido: $27 / 172016$

Aceptado: 28/03/207

\author{
Antonio Ruiz-Porras ${ }^{1}$ \\ antoniop@cucea.udg.mx \\ Juan Carlos Zagaceta-García ${ }^{2}$ \\ jczagaceta@iteso.mx
}

\section{Resumen}

Este estudio examina y compara la relación entre la tercerización económica y el crecimiento de la productividad agregada para economías en diferentes niveles de desarrollo. El estudio usa evaluaciones shift-share y estimaciones econométricas en panel. El análisis shift-share se usa para descomponer la productividad agregada por sectores económicos. Los modelos econométricos evalúan la relación entre la tercerización económica y el crecimiento de la productividad agregada. Usamos datos para siete economías desarrolladas y doce en desarrollo.

Palabras Clave: Desarrollo económico, econometría de panel, productividad, servicios, terciarización

\section{Abstract}

This study examines y compares the relationship between economic tertiarization and aggregated productivity growth for economies in different stages of development. The study uses shift-share assessments and estimations for paneldata. The shift-share analysis is used to decompose the aggregate productivities by economic sectors. The econometric models assess the relationship between economic tertiarization and aggregated productivity growth. We use data for seven developed economies and twelve developing ones.

Keywords: Economic development, panel-data econometrics, productivity, services, tertiarization

1 Departamento de Métodos Cuantitativos. Universidad de Guadalajara, CUCEA. Periférico Norte 799, Núcleo Universitario Los Belenes, 45100, Zapopan, Jalisco, México.

2 Centre Lillois d'Etudes et de Recherches Sociologiques et Economiques (Clersé). Université de Lille 1. Cité Scientifique. 59655. Villeneuve d'Ascq. Cedex. France. 


\section{Introducción}

En este estudio se analiza la productividad laboral y, en particular, la correspondiente al sector terciario para economías desarrolladas y en desarrollo. Su relevancia se plantea en términos de la necesidad de entender los procesos de desarrollo económico. El estudio utiliza técnicas de estadística descriptiva, de análisis shift-share y de regresión para paneles de datos con efectos aleatorios. Particularmente, la técnica shift-share se utiliza para estudiar las relaciones existentes entre los sectores económicos, los servicios y la productividad laboral con base en la metodología de Maroto y Cuadrado (2011). Las regresiones, por su parte, se utilizan para estudiar los determinantes del crecimiento de la productividad laboral en los subsectores del sector terciario. La motivación de esta investigación se vincula a la escasa literatura que ha analizado a los servicios como determinantes de la productividad y del desarrollo económico. ${ }^{3}$

Las principales preguntas que guían esta investigación son las siguientes: 1) ¿Cómo ha evolucionado la productividad agregada en las economías desarrolladas y en desarrollo?; 2) ¿Cuál ha sido el papel del sector terciario y los subsectores del sector servicios dentro de dichas transformaciones?; 3) ¿Cómo se caracterizan las relaciones entre la terciarización económica y el crecimiento de la productividad agregada? ; 4) ¿Hay patrones similares o disímiles entre las economías desarrolladas y en desarrollo?

La investigación se justifica por razones de política económica y de tipo académico. Particularmente, aquí se estudian los servicios porque se ha argumentado que los mismos han definido los procesos de desarrollo contemporáneos (Peneder, et. al., 2003). Su estudio también se justifica porque los servicios generan hasta el 70 por ciento de la producción y del empleo en algunas economías. El interés por incluir economías en desarrollo se justifica porque la mayoría de los estudios se enfocan en las economías desarrolladas. ${ }^{4}$ De hecho, este estudio es uno de los primeros que utiliza la técnica shift-share para estudiar los servicios para economías en desarrollo.

3 Hay varios estudios que analizan el sector secundario y las manufacturas . Entre estos estudios se incluyen aquellos de Pavcnik (2000), Paus, et al. (2003), Cavalcanti-Ferreira y Rossi (2003), Fernandes (2007) y Esclava, et al. (2009).

4 Algunos estudios representativos para economías desarrolladas incluyen los de Stigler (1956), Salter (1960), Maddison (1980), Gershuny y Miles (1983), Howe (1986), Kutscher y Personick (1986). 
Metodológicamente, la investigación utiliza técnicas complementarias y relativamente independientes entre sí. Particularmente, la estadística descriptiva se utiliza para mostrar las similitudes y diferencias entre las economías desarrolladas y en desarrollo. La técnica de shift-share se usa para mostrar las relaciones entre los sectores económicos, los servicios y la productividad laboral. Las técnicas de regresión para paneles de datos, por su parte, se utilizan para estimar los impactos que tienen los servicios, tanto a nivel agregado y desagregado, sobre el crecimiento de la productividad laboral.

El estudio se divide en cinco secciones: en la segunda sección se revisa la literatura, en la tercera sección se plantean y justifican las técnicas y métodos de análisis, en la cuarta sección se muestran las estimaciones de estadística descriptiva, del análisis shift-share y del análisis econométrico y en la quinta sección se sintetizan los resultados, se discuten sus implicaciones y se plantean algunas líneas de investigación futura.

\section{Revisión de la literatura}

En las últimas décadas, la producción de servicios ha ganado relevancia en la economía global. Según algunas estimaciones, los servicios proveen aproximadamente el $70 \%$ del PIB y del empleo total en las economías desarrolladas. Por esta razones, los servicios han permitido transformar la producción y el empleo, de tal modo, que ahora se acepta que los servicios constituyen el tercer sector de la economía . La "terciarización", por tanto, es el proceso de transformación de las economías como consecuencia del crecimiento de la importancia de los servicios (Peneder, et al., 2003).

Usualmente, se plantea que la terciarización incrementa la productividad y, eventualmente, que contribuye al desarrollo de las economías. Las teorías que plantean este argumento suelen indicar que la terciarización genera cambios en: 1) las tecnologías industriales; 2) la demanda agregada; y, 3) los patrones de comercio internacional. Los mencionados cambios permiten redistribuir los factores de producción en los niveles micro y macro. Más aún, permiten que los factores puedan ser empleados de maneras más eficientes en las economías. Por 
tanto, lo que el argumento plantea es que la terciarización permite incrementar la productividad agregada y por añadidura, el crecimiento económico porque facilita la re asignación de los factores de la producción (Kuznets, 1966; Hsieh y Klenow, 2009; Dabla-Norris et al., 2013). Así, y con base en la argumentación planteada, hay quienes creen que los procesos de terciarización debieran ser incentivados para promover el desarrollo económico.

Las teorías mencionadas han sido objeto de críticas por parte de algunos académicos y hacedores de políticas. Más aún, hay quienes indican que es necesario desincentivar los procesos de terciarización económica. Particularmente, los teóricos que argumentan en contra de la terciarización suelen señalar que los servicios tienen una baja productividad que limita las posibilidades de desarrollo de las economías (Fourastié, 1949; Baumol 1967). Su argumento plantea que la baja productividad de los servicios se refleja en salarios bajos, una baja capacidad de ahorro y una baja capacidad de inversión productiva. Además, hay quienes extienden el argumento a fin de plantear que la terciarización y la baja productividad de los servicios inhiben el crecimiento de las economías. Todas estas ideas se sintetizan en la "Teoría de la enfermedad de los costos" (cost-disease theory).

Las controversias relativas a unas y otras teorías han motivado el desarrollo de investigación empírica. En este contexto, debe reconocerse que hay escasa evidencia que valide la teoría de la enfermedad de los costos. Incluso, Baumol (2002) identifica ciertos tipos de servicios, como los basados en la innovación y el conocimiento, que contradicen a la mencionada teoría. Más aún, hay estudios que sugieren que existen sub-sectores de los servicios muy productivos, como los relacionados con la información y las comunicaciones (O’Mahony y Ark, 2003, Stiroh, 2002). Sin embargo, debe reconocerse que la teoría de la enfermedad de los costos todavía tiene algunos seguidores. Todavía no hay consensos sobre las relaciones empíricas entre el sector terciario y el desarrollo económico.

El debate empírico no es concluyente por varias razones. Particularmente hay quienes argumentan que la terciarización induce cambios estructurales que perjudican las expectativas de industrialización de las economías. Sus argumentos se sustentan en los estudios de Fisher (1935), Clark (1940), 
Fourastié (1949), y Kuznets (1957). En estos estudios se plantea que los cambios estructurales inducen caídas y aumentos de la producción y el empleo que, bajo algunas circunstancias, pueden perjudicar el crecimiento de las economías. Particularmente, Feinstein (1999) muestra que después de los años setentas, las economías desarrolladas experimentaron aumentos en el empleo manufacturero y de servicios y caídas en el empleo agrícola. También muestra que el sector terciario creció más rápidamente que los otros sectores. Fisher (1939), Stigler (1956), Mazumdar (1976) y Gemmell (1982) explican estos cambios indicando que cuando los ingresos personales se incrementan, el gasto en comida tiende a bajar y por extensión, la demanda de productos agrícolas; reduciéndose así la participación del sector primario y aumentando al mismo tiempo la demanda de servicios. En este contexto, no sobra mencionar que, en los años setentas, las economías desarrolladas experimentaron procesos estanflacionarios.

Hay estudios para economías en vías de desarrollo que validan que el crecimiento del empleo y la producción ocurren para unos sectores económicos en detrimento de otros. Particularmente, Ramos (1970) y Squire (1979), encuentran que el empleo en el sector de servicios crece más rápidamente en las economías en desarrollo que en las economías desarrolladas. Más aún, ellos hallan que varias economías latinoamericanas transitaron del sector primario al terciario, sin haberse transformado en economías manufactureras. Además, Squire (1979) halla que los servicios que caracterizan a las economías en desarrollo son aquellos de baja productividad. Por tanto, los efectos de la terciarización resultan un tanto ambiguos.

La importancia de promover el sector terciario en las economías en desarrollo también ha sido cuestionada por razones metodológicas. La falta de datos desagregados para dichas economías ha hecho difícil validar las teorías existentes (elaboradas, en su mayoría, en el contexto de las economías desarrolladas). Pandit (1989), por ejemplo, señala que los estudios que vinculan el crecimiento del sector terciario con la creación de empleo han sido poco satisfactorios. Asimismo, hay quienes priorizan que la hipótesis de que la salida del subdesarrollo solo puede lograrse impulsando el sector secundario y, en particular, la producción de manufacturas (Nurkse, 1955). Por estas razones, todavía hay controversias sobre las políticas que debieran adoptarse con respecto al sector terciario en las economías en desarrollo. 
Una limitación metodológica de los estudios empíricos estriba en que los servicios no son fáciles de clasificar y analizar. Los servicios son muy heterogéneos y diversos y, por tanto, resultan muy complejos. Para resolver esta limitación, Johnston (1975) propuso una clasificación basada en la manera en que participan los recursos humanos en la oferta de servicios. Así, Johnston clasifica a los servicios en "market services" y en "no market services". Particularmente, los servicios "market services" son aquellos que se ofrecen para su venta en los mercados, mientras que los servicios "no market services" son aquellos que son ofrecidos por los gobiernos y comunidades de manera subsidiada o sin fines de lucro (seguridad, salud, educación). En esta clasificación explícitamente se asume que los servicios 'market services' incrementan la productividad de los trabajadores y la economía; mientras que los servicios 'no market services' no lo hacen. ${ }^{5}$ Estos supuestos, si bien un tanto controversiales, son comúnmente adoptados en la literatura empírica (Maroto y Cuadrado, 2011).

Finalmente no sobra enfatizar que las relaciones entre el sector terciario, la productividad y el desarrollo económico constituyen un campo promisorio para realizar investigación. Particularmente, las diversas teorías, los debates sin resolver y las limitaciones metodológicas validan la pertinencia de hacer estudios para las economías en desarrollo. Estas consideraciones motivan la investigación aquí planteada. Por estas razones, en las siguientes secciones se plantean la metodología y las técnicas utilizadas en la investigación; y se presentan y discuten los resultados obtenidos. Ello con fines de facilitar enmarcar la investigación dentro de la literatura existente.

\section{Materiales y métodos}

En esta investigación se usan las economías y los años como unidades de medición y de tiempo, respectivamente. Particularmente, los indicadores utilizados son construidos usando datos anuales desagregados a nivel sectorial de siete economías desarrolladas y de doce economías en desarrollo (i.e., diecinueve economías en total). Las primeras incluyen a Alemania, Canadá, Estados Unidos, Francia, Italia,

5 La teoría microeconómica muestra que, en ausencia de distorsiones, la productividad laboral se mueve de manera similar a los costos de la mano de obra. Por esta razón, también se asume que las variaciones de precios de los servicios "Market services" suelen ser menos abruptas que aquellas de los servicios 'No market services'. Bhagwati (1984) e Inklaar y Timmer (2014) señalan que existe cierta evidencia de que los servicios intensivos en mano de obra experimentan grandes variaciones en sus precios. 
Japón y Reino Unido. Las economías en desarrollo incluyen a Brasil, Eslovenia, Estonia, Filipinas, Hungría, Indonesia, Malasia, México, Polonia, República Checa, República Eslovaca y Turquía. ${ }^{6}$ Por conveniencia metodológica, los servicios se clasifican con base en la propuesta de Johnston (1975). Esta clasificación se usa para facilitar la comparabilidad de la investigación con otros trabajos sobre el tema. ${ }^{7}$ El periodo de cobertura de los datos abarca el periodo entre 2004 y 2009.

Tabla 1: Clasificación sectorial económica

\begin{tabular}{ll}
\hline SECTOR & DESCRIPCIÓN \\
\hline Manufactura & Divisiones 10-37 \\
\hline & a) 'Market services' (divisiones 50-74) \\
& a.1) Comercio al por Mayor y al por Menor; Reparaciones; Hoteles y Restaurantes; Transportes (divisiones 50-64) \\
Servicios & a.2) Establecimientos Financieros; Bienes Inmuebles y Servicios prestados a las Empresas (divisiones 65-74) \\
& b) 'No market services' (divisiones 75-97) \\
& Administración Pública, Enseñanza, Servicios Sociales y de Salud, Servicios Domésticos \\
\hline 'Resto' & a) Agricultura (divisiones 1-5) \\
& b) Construcción (división 45)
\end{tabular}

Fuente: Elaboración propia con base en CIIU Rev. 3 (Clasificación Industrial Internacional Uniforme, Revisión 3).

Notas: Algunas economías tienen de orgen la información en otros clasificadores: SCIAN (Sistema de Clasificación Inustril de América del Norte), Clasificación Industrial Estándar de Malasia y NACE (Clasificación Estadística de las Actividades Económicas en la Comunidad Eropea) Rev. 2.

En la Tabla 1, se muestra la clasificación desagregada usada para comparar los sectores primario, secundario y terciario con la productividad global. Esta tabla, además, permite visualizar el aporte económico de los diferentes tipos de servicios. El clasificador industrial utilizado para desagregar los sectores económicos es el CIIU Rev.3 (Clasificación Industrial Internacional Uniforme, Revisión 3). ${ }^{8}$

6 Las fuentes de datos utilizadas para este análisis (denominadas como fuentes base) son las siguientes: Banco Mundial, OCDE, CEPAL, SEDLAC, Instituto Brasileño de Geografía y Estadística, Oficina Nacional de Estadísticas de Filipinas, Banco Asiático de Desarrollo, Estadísticas de Indonesia, Departamento de Estadísticas de Malasia, Instituto de Estadística Turco y la Agencia Nacional de Estadísticas de Canadá.

7 Véase, entre otros, los trabajos de Maroto y Cuadrado, 2007 y 2011; Timmer y de Vries, 2009; e Inklaar y Timmer, 2014.

8 En el Apéndice se muestra la Clasificación Industrial Internacional Uniforme (Rev. 3.1) de las actividades económicas. 


\subsection{Análisis shift-share}

El análisis shift-share se usa para mostrar las relaciones entre los sectores económicos, los servicios y la productividad agregada en las diecinueve economías analizadas. El análisis se sustenta en el supuesto de que el crecimiento de la productividad laboral ocurre debido a dos efectos complementarios entre sí: El "efecto within" y el "efecto shift". Particularmente, el "efecto within" es el crecimiento de la productividad que ocurre por la acumulación de capital, por el cambio tecnológico o por la minimización de la localización inadecuada de fábricas. El "efecto shift", por su parte, es el crecimiento que ocurre cuando los trabajadores se mueven de los sectores de baja productividad a los de alta productividad. El "efecto shift" también suele denominarse como el "efecto estructural".

El análisis se sustenta en la técnica shift-share. Esta técnica tiene algunas características que deben señalarse para efecto de definir los alcances y límites de este estudio. La principal característica es que requiere de relativamente pocos datos para ser implementada (Knudsen, 2000). Esta característica facilita la implementación de la técnica en las economías en desarrollo y facilita la comparabilidad entre las economías desarrolladas y en desarrollo. Otra característica es que la técnica supone que no hay relaciones causales entre las variables (Temple y Woessmann 2006; Timmer y de Vries, 2009). Por tanto, la técnica no requiere ni puede distinguir entre las causas y las consecuencias de la terciarización. ${ }^{9}$

La metodología utilizada aquí sigue la propuesta de Maroto y Cuadrado, (2011). En dicha propuesta, ellos descomponen el crecimiento de la productividad global en los efectos 'within' y "shift". El "efecto shift", además, se descompone en un 'efecto estático' y en un 'efecto dinámico'. La ventaja de descomponer el "efecto shift" estriba en que el mismo puede analizarse tanto en términos del cambio en la participación laboral (i.e., el efecto estático), como en términos de la manera en que dicha participación influye en la productividad laboral (i.e., el efecto dinámico).

9 Temple y Woessmann (2006) y Timmer y de Vries (2009) argumentan que este problema de endogeneidad estructural ocurre debido a variaciones de la demanda, de los flujos comerciales y de los precios globales. 
Las principales fórmulas utilizadas para el análisis shift-share son las siguientes:

$$
\begin{aligned}
& \text { Tasa de crecimiento de la productividad laboral } \quad=\frac{\pi_{t}}{\pi_{t-n}} \\
& =\text { Efecto estático }+ \text { Efecto dinámico }+ \text { Efecto Within } \\
& \text { Productividad laboral } \\
& \text { Efecto estático } \\
& =\frac{\sum_{i=1}^{N} \pi_{i, t} s_{i, t}-s_{i, t-n}}{\pi_{t-n}} \\
& =\frac{\sum_{i=1}^{N} \pi_{i, t}-\pi_{i, t-n} s_{i, t}-s_{i, t-n}}{\pi_{t-n}} \\
& =\frac{\sum_{i=1}^{N} \pi_{i, t-} \pi_{i, t-n} s_{i, t}}{\pi_{t-n}} \\
& S_{i}=\frac{L_{i}}{L}
\end{aligned}
$$

donde: $\pi$ es la productividad laboral ${ }^{10} t-n$ es el año inicial; $t$ es el año final; el subíndice $i$ define los sectores económicos, y $S$ es la participación del sector en términos del empleo.

Metodológicamente, el análisis shift-share comprende tres etapas. En la primera etapa se hace la estadística descriptiva de los indicadores de las economías desarrolladas y en desarrollo, la estadística descriptiva se hace para los tres sectores económicos considerados aquí (Manufactura, Servicios y 'Resto'). En la segunda etapa se estiman los efectos within y shift para los tres sectores económicos, diferenciando entre los efectos estimados para las economías desarrolladas y los estimados para las economías en desarrollo. En la tercera etapa se estiman los efectos within y shift para los subsectores que integran el sector terciario. Así se evalúan los impactos que tienen en la productividad laboral los servicios clasificados como "Market services" y "No market services".

El análisis shift-share, tal como se ha planteado, permite cuantificar las contribuciones que tienen los sectores económicos en la productividad laboral. En este contexto, vale la pena señalar que los estudios hechos para Europa y los EEUU concluyen que el efecto 'within' domina al efecto 'shift' ${ }^{11}$. Sin embargo, los

10 La productividad laboral se calculó a partir de dividir el PIB entre el número de personas que laboran para cada sector.

11 Véase Maroto y Cuadrado (2007, 2009 y 2011), Peneder et.al. (2003), Faberger (2000) y Timmer y Szirmai (2000). 
escasos estudios existentes para economías en desarrollo no siempre validan dicho hallazgo. ${ }^{12}$ Por esta razón, el análisis se utiliza para evaluar si se cumple o no dicho hallazgo para las economías analizadas. Además, el análisis se utiliza para evaluar la hipótesis de que los servicios tienen una contribución negativa en la productividad laboral. ${ }^{13}$ Esta evaluación es necesaria para aquellos que discuten la pertinencia del sector terciario en el desarrollo económico. De hecho, su validez implicaría que no sería pertinente inducir la terciarización de las economías con fines de promover su crecimiento económico.

\subsection{Análisis econométrico}

El análisis econométrico se utiliza aquí para estimar los impactos que tienen los servicios, tanto a nivel agregado y desagregado, sobre el crecimiento de la productividad laboral. Particularmente, el análisis se sustenta en estimaciones de regresiones para paneles con efectos aleatorios. El uso de efectos aleatorios supone que las relaciones de las variables no están definidas por las particularidades de las economías. Además, en todas las regresiones se utiliza el crecimiento de la productividad laboral como la variable dependiente. Las variables independientes utilizadas incluyen varios indicadores de la participación de los servicios en los mercados de trabajo. Las variables de control refieren a los determinantes tradicionales del crecimiento económico. ${ }^{14}$ La muestra de indicadores analizados incluye diecisiete economías. Se excluyen los indicadores de las economías de Brasil y Malasia debido a la falta de los datos necesarios para construir las variables de control.

El análisis econométrico se sustenta en la estimación de tres grupos de regresiones, estos grupos se definen en términos del tipo de servicios analizados. Particularmente, el grupo denominado "servicios" incluye estimaciones que utilizan indicadores del sector terciario a nivel agregado. El grupo denominado "Market services", por su parte, incluye estimaciones

12 Timmer y de Vries (2009) analizan economías latinoamericanas y asiáticas.

13 Véase Baumol (1967) y Fourastié (1949).

14 Los estudios sectoriales sobre los determinantes del crecimiento económico suelen incluir variables referidas a la creación de capital, la educación, la composición de la población, la apertura comercial y la aplicación del conocimiento. Véase, entre otros, a Denison (1967), Chung y Denison (1976), Dabla-Norris et al. (2014) y Wolszczak-Derlacz 2014). 
para servicios que se venden en los mercados. El grupo denominado "No market services" incluye estimaciones para servicios que se ofrecen de manera subsidiada o sin fines de lucro. Cada grupo de regresiones incluye tres estimaciones: la primera estimación incluye indicadores de las economías en desarrollo, la segunda incluye indicadores de las economías desarrolladas y la tercera estimación incluye indicadores de ambos tipos de economías. Así, los mencionados grupos de regresiones se utilizan para analizar los determinantes de la productividad laboral considerando los diferentes tipos de servicios y de economías.

Metodológicamente, las regresiones se estiman asumiendo ciertos supuestos. Particularmente, todas las estimaciones se realizan considerando una forma funcional semilogarítmica (lin-log). En dicha forma funcional, los coeficientes estimados muestran los cambios absolutos ocurridos en la variable dependiente debidos a cambios porcentuales de las variables independientes. Por tanto, los coeficientes equivalen a las semi-elasticidades (i.e., las variaciones en la productividad laboral debidas a un incremento de la participación de los servicios en el mercado de trabajo). Así, el modelo de regresión para datos en panel utilizado para hacer las estimaciones es el siguiente:

$$
\Delta \pi_{i}=\alpha+\beta \ln \pi_{i, t-n}+\gamma \Delta \ln s_{i+} \delta \ln s_{i, t-n+} \phi \ln Z_{i, t}+v_{i}+\varepsilon_{i, t}
$$

donde $i=1,2, \ldots, K$ son las economías, $t$ es la longitud del periodo considerado; $s_{i}$ es la participación laboral del sector (sobre el total del personal empleado) en la economía $i ; \Delta \pi_{i}$ es el índice de crecimiento de la productividad laboral; $Z_{i}$ es la matriz de variables auxiliares de control; $v_{i}$ es el componente del error de los efectos aleatorios; y $\varepsilon_{i, t}$ el residuo del modelo.

La validación econométrica de las regresiones se realiza utilizando diversas pruebas estadísticas. Particularmente, la prueba de Jarque-Bera se utiliza para validar el supuesto de normalidad de los residuales. La significancia individual de las variables se evalúa usando pruebas basadas en el estadístico z. La significancia conjunta de las variables se evalúa usando pruebas basadas en el estadístico de Wald. 
La productividad inicial y la participación laboral inicial de los sectores o subsectores para cada clasificación de economías, se utilizan como parámetros de referencia en todas las estimaciones. Por esta razón se prefiere usar la técnica con 'efectos aleatorios' en las regresiones. La conveniencia de usar dicha técnica se sustenta en la consideración de que los efectos fijos son redundantes cuando hay parámetros de referencia en las estimaciones. En este contexto, debe señalarse que los modelos estimados con la técnica de "efectos aleatorios" tienen ciertas limitaciones: primeramente, el error idiosincrático, $\varepsilon$, no es observable directamente, asimismo, las estimaciones requieren el cumplimiento de ciertos supuestos estadísticos sobre las perturbaciones (dado que incluyen errores idiosincráticos y errores de las unidades de medición). ${ }^{15}$

El análisis econométrico se complementa con el análisis de los signos esperados. Este último análisis se hace para validar las hipótesis sobre las relaciones entre los servicios y la productividad. Particularmente, y con base en consideraciones teóricas, se espera que las variables vinculadas al "efecto shift" y las variables de control sean significativas y que los coeficientes estimados sean positivos. También se espera que los coeficientes estimados para los servicios 'Market services' sean positivos; mientras que los coeficientes para los servicios 'No market services' sean negativos o no significativos. Además, se esperaría que el signo de la productividad inicial del sector o sub-sector fuera negativo por concordancia con la teoría de la convergencia condicional. ${ }^{16}$

\section{Análisis de las estimaciones}

En esta sección, se muestran las estimaciones del análisis de estadística descriptiva, del análisis shift-share y del análisis econométrico del panel de datos.

\footnotetext{
15 Véase Wooldridge (2010) para una introducción a las técnicas econométricas para paneles de datos.

16 Contrario al modelo de crecimiento económico de Solow-Swan de convergencia absoluta, el cual, postula que las economías pobres tienden a crecer per cápita más deprisa que las economías ricas cuando no están condicionas por alguna característica de las mismas. La convergencia condicional, permite la existencia de heterogeneidad entre las economías, y en este caso una economía crece más rápido cuanto más lejos se encuentra de su propio estado estacionario (Barro y Sala-i-Martin, 2004).
} 


\subsection{Estimaciones de estadística descriptiva}

Aquí se analiza la estadística descriptiva de los indicadores utilizados en el análisis shift-share y econométrico. Particularmente, la Tabla 2 muestra la estadística descriptiva de la variable participación laboral y de los índices de productividad. La Tabla 3 muestra la estadística descriptiva de las variables de control usadas en el análisis econométrico. Las variables de control incluyen indicadores de apertura comercial (exportaciones de bienes y servicios + importaciones de bienes y servicios / PIB); de capital físico (formación bruta de capital fijo como $\%$ del PIB); de capital humano (matrícula de educación primaria + matrícula de educación secundaria / índice del personal empleado mayor de 15 años y la población total); y de composición demográfica (índice del personal empleado mayor de 15 años / población total).

La Tabla 2 muestra que la participación de los servicios y la productividad laboral son mayores en las economías desarrolladas que en las economías en desarrollo. Las estimaciones muestran que, en promedio, la participación del sector terciario es diecinueve puntos porcentuales más grande en las economías desarrolladas que en las economías en desarrollo. Particularmente, los subsectores que más contribuyen a esta diferencia son los 'No market services' y de los establecimientos financieros, inmobiliarias y servicios a empresas. Las estimaciones también muestran que la productividad en las economías desarrolladas es 3.24 veces más alta que en sus contrapartes en desarrollo. 
Tabla 2: Estadística descriptiva de la participación laboral y del índice de productividad (19 economías, 2004-2009

\begin{tabular}{|c|c|c|c|c|c|}
\hline & \multicolumn{3}{|c|}{ PARTICIPACIÓN LABORAL } & \multirow[b]{2}{*}{ Minimo } & \multirow[b]{2}{*}{ Máximo } \\
\hline & Observaciones & Media & Desviación estándar & & \\
\hline \multicolumn{6}{|l|}{ ECONOMIASSEN DESARROLLO } \\
\hline \multicolumn{6}{|l|}{ Sectores económicos } \\
\hline Manufactura & 72 & 0.2115 & 0.0590 & 0.0880 & 0.2951 \\
\hline Servicios & 72 & 0.5571 & 0.0675 & 0.3763 & 0.6522 \\
\hline Resto & 72 & 0.2314 & 0.1127 & 0.1141 & 0.4896 \\
\hline \multicolumn{6}{|l|}{ Sector servicios des agregado } \\
\hline \multicolumn{6}{|l|}{ Market services: } \\
\hline $\begin{array}{l}\text { Comercio al por mayor y al por menor; reparaciones; } \\
\text { hoteles y restaurantes; transportes. }\end{array}$ & 72 & 0.2691 & 0.0236 & 0.2221 & 0.3420 \\
\hline $\begin{array}{l}\text { Establecimient os financieros; bienes inmuebles y } \\
\text { servicios prestados a las empresas. }\end{array}$ & 72 & 0.0374 & 0.0179 & 0.0116 & 0.0773 \\
\hline No market services & 72 & 0.2506 & 0.0620 & 0.1107 & 0.3386 \\
\hline \multicolumn{6}{|l|}{$\begin{array}{r}\text { ECONOMIAS DESARROLLADAS } \\
\end{array}$} \\
\hline \multicolumn{6}{|l|}{ Sectores económicos } \\
\hline Mamufactura & 42 & 0.1515 & 0.0396 & 0.0949 & 0.2128 \\
\hline Servicios & 42 & 0.7477 & 0.0516 & 0.6683 & 0.8212 \\
\hline Resto & 42 & 0.1008 & 0.0211 & 0.0747 & 0.1486 \\
\hline \multirow{2}{*}{\multicolumn{6}{|c|}{$\begin{array}{l}\text { Sector servicios des agr egado } \\
\text { Market services: }\end{array}$}} \\
\hline & \multicolumn{5}{|c|}{ Market services: } \\
\hline $\begin{array}{l}\text { Comercio al por mayor y al por menor; reparaciones; } \\
\text { hoteles y restaurantes; transportes. }\end{array}$ & 42 & 0.2800 & 0.0315 & 0.2414 & 0.3468 \\
\hline $\begin{array}{l}\text { Establecimient os financieros; bienes inmuebles y } \\
\text { servicios prestados a las empresas. }\end{array}$ & 42 & 0.0808 & 0.0640 & 0.0295 & 0.1931 \\
\hline \multirow[t]{3}{*}{ No market services } & 42 & 0.3869 & 0.0682 & 0.2540 & 0.4764 \\
\hline & \multicolumn{3}{|c|}{ INDICE DE PRODUCTIVIDAD } & & \\
\hline & Observaciones & Media & Desviación estándar & Minimo & Máximo \\
\hline \multicolumn{6}{|l|}{ ECONOMIAS EN DESARROLLO } \\
\hline \multicolumn{6}{|l|}{ Sectores económicos } \\
\hline Mamufactura & 72 & 2.3945 & 0.9970 & 0.7017 & 4.6888 \\
\hline Servicios & 72 & 2.1431 & 1.0882 & 0.3077 & 4.5302 \\
\hline Resto & 72 & 1.3004 & 0.8352 & 0.1296 & 3.8221 \\
\hline \multicolumn{6}{|l|}{ Sector servicios des agregado } \\
\hline \multicolumn{6}{|l|}{ Market services: } \\
\hline $\begin{array}{l}\text { Comercio al por mayor y al por menor; reparaciones; } \\
\text { hoteles y restaurantes; transportes. }\end{array}$ & 72 & 1.9835 & 1.0851 & 0.2478 & 4.3124 \\
\hline $\begin{array}{l}\text { Establecimient os financieros; bienes inmuebles y } \\
\text { servicios prestados a las empresas. }\end{array}$ & 72 & 9.0595 & 7.9365 & 0.5009 & 37.9320 \\
\hline No market services & 72 & 1.5627 & 0.8232 & 0.2413 & 3.4748 \\
\hline \multicolumn{6}{|l|}{$\begin{array}{r}\text { ECONOMIAS DESARROLLADAS } \\
\end{array}$} \\
\hline \multicolumn{6}{|l|}{ Sectores económicos } \\
\hline Mamufactura & 42 & 9.4017 & 1.7916 & 6.2512 & 13.3494 \\
\hline Servicios & 42 & 6.9371 & 1.3108 & 4.1117 & 11.0382 \\
\hline Resto & 42 & 4.8780 & 0.9505 & 3.0687 & 6.7699 \\
\hline \multicolumn{6}{|l|}{ Sector servicios des agregado } \\
\hline \multicolumn{6}{|l|}{ Market services: } \\
\hline $\begin{array}{l}\text { Comercio al por } m \text { ayor } y \text { al por menor; reparaciones; } \\
\text { hoteles y restaurantes; transportes. }\end{array}$ & 42 & 6.0390 & 2.1032 & 2.9436 & 16.8760 \\
\hline $\begin{array}{l}\text { Establecimient os financieros; bienes inmuebles y } \\
\text { servicios prestados a las empresas. }\end{array}$ & 42 & 24.6999 & 10.2375 & 6.8200 & 42.9046 \\
\hline No market services & 42 & 5.2875 & 0.6400 & 3.7697 & 6.5124 \\
\hline
\end{tabular}

Fuente: Elaboración propia

Nota: El sector 'Resto' está compuesto por los sectores de la agricultura (divisiones 1-5) y construcción (división 45) 
Tabla 3: Estadística descriptiva de las variables de control (17 economías, 2005-2009)

\begin{tabular}{lccccc}
\hline & Observaciones & Media & Desviación estándar & Mínimo & Máximo \\
\hline ECONOMÍAS EN DES ARROLLO & & & & & \\
\hline Apertura comercial & 60 & 1.2692 & 0.5635 & 0.4937 & 2.5257 \\
Capital fisico & 60 & 24.9310 & 4.9853 & 14.9377 & 38.6968 \\
Capital humano & 60 & 3.6645 & 0.4624 & 2.8526 & 4.5201 \\
Composición demográfica & 60 & 53.3050 & 6.0998 & 41.8000 & 62.2000 \\
\hline ECONOMÍAS DESARROLLADAS & & & & 0.2269 & 0.9479 \\
\hline Apertura comercial & 42 & 0.5734 & 0.2261 & 14.0960 & 23.2397 \\
Capital fisico & 42 & 19.8780 & 2.4060 & 1.5720 & 4.5094 \\
Capital humano & 42 & 3.6876 & 0.5642 & 44.8000 & 63.2000 \\
Composición demográfica & 42 & 55.7690 & 5.6838 &
\end{tabular}

Fuente: Elaboración propia

Nota: Las economías de Brasil y Malasia fueron excluidas por la falta de disponibilidad de datos

La Tabla 3 muestra que hay resultados mixtos cuando se comparan las variables de control. Particularmente, las estimaciones de las medias muestran que en las economías en desarrollo hay una mayor apertura comercial y una mayor inversión en capital físico que en sus contrapartes desarrolladas. Las estimaciones también muestran que las economías en desarrollo tienen menos capital humano y una población trabajadora relativamente más joven que en sus contrapartes desarrolladas. Las estimaciones de las desviaciones estándar, por su parte, muestran que los indicadores de las economías en desarrollo son más heterogéneos que los correspondientes a las economías desarrolladas.

La evidencia sugiere que ha habido un proceso de terciarización para todas las economías analizadas. Particularmente, en las economías en desarrollo, la participación de los servicios en los mercados de trabajo ha sido creciente durante el periodo estudiado. Sin embargo, no sobra señalar, que la mayor terciarización ha reducido la participación de los otros sectores de las economías. Pese a ello, también debe señalarse que la agricultura, la ganadería, la producción de bienes, la extracción de materias primas y la producción de energía, entre otras, seguirán siendo importantes en la economía global. En este contexto, cabe señalar que hay quienes argumentan que la terciarización de las economías y los cambios en la participación de los sectores primario y secundario podrían reflejar mejoras de los procesos productivos. ${ }^{17}$ Si dicha mejora ocurriera, el desarrollo del sector terciario podría incrementar indirectamente la productividad de las economías.

17 Dichas argumentaciones se sintetizan en World Bank (2007). 


\subsection{Estimaciones de la técnica shift-share}

El análisis shift-share se utiliza para estudiar las relaciones existentes entre los sectores económicos, los servicios y la productividad laboral. El mismo se sustenta en estimaciones de los efectos "within" y "shift" para las economías desarrolladas y en desarrollo. ${ }^{18}$ Por simplicidad, las tablas de resultados muestran únicamente los promedios de dichas estimaciones. Particularmente, la Tabla 4 muestra los promedios considerando los indicadores agregados de los tres sectores económicos y la productividad laboral. La Tabla 5, por su parte, muestra los promedios estimados considerando los indicadores desagregados de los subsectores del sector servicios y la productividad.

Las tablas proveen información complementaria sobre las relaciones analizadas en los sectores económicos y los subsectores del sector servicios. Así las tablas muestran los promedios de la productividad laboral y de la participación de los sectores y subsectores en la producción, en los mercados de trabajo y en la productividad laboral. Específicamente, las tablas muestran: 1) las tasas de crecimiento de la productividad laboral, 2) las proporciones de la producción de los sectores y subsectores con respecto al PIB, 3) las proporciones del empleo en los sectores y subsectores con respecto a la fuerza de trabajo y 4) las proporciones de la productividad laboral de los sectores y subsectores con respecto a la productividad laboral agregada.

Tabla 4: Análisis de la descomposición del crecimiento de la productividad laboral

(19 economías, 2005.2009)

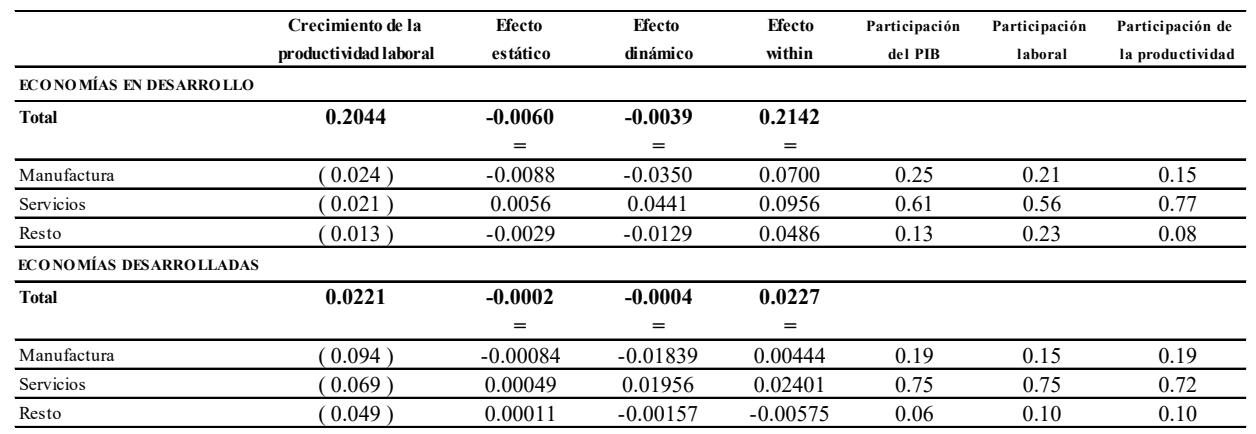

Fuente: Elaboración propia

Nota: 'Resto' está compuesto por los sectores de la agricultura (divisiones 1-5) y construcción (división 45)

18 Adviértase que el "efecto shift" se integra por los efectos estático y dinámico. 
La Tabla 4 muestra que el crecimiento de la productividad laboral a nivel agregado ha dependido en gran medida del "efecto within". Esto significa que la re localización laboral entre los sectores económicos de alta y baja productividad, i.e. "el efecto shift", tiene escasa importancia para incrementar la productividad. En este contexto, no sobra señalar que las estimaciones muestran que el "efecto shift" contribuye, marginalmente, al crecimiento de la productividad en el sector terciario. Estos hallazgos son válidos tanto para las economías desarrolladas como para sus contrapartes en desarrollo. Por tanto, hay alguna evidencia a favor de que la reubicación de los trabajadores en el sector terciario incrementa la productividad laboral de las economías.

Tabla 5: Análisis de la descomposición del crecimiento de la productividad laboral para el sector terciario (19 economías, 2005-2009)

\begin{tabular}{|c|c|c|c|c|c|c|c|}
\hline & $\begin{array}{c}\text { Crecimiento de la } \\
\text { productividad laboral }\end{array}$ & $\begin{array}{l}\text { Efecto } \\
\text { estático }\end{array}$ & $\begin{array}{c}\text { Efecto } \\
\text { dinámico }\end{array}$ & $\begin{array}{l}\text { Efecto } \\
\text { within }\end{array}$ & $\begin{array}{l}\text { Participación } \\
\text { del PIB }\end{array}$ & $\begin{array}{c}\text { Participación } \\
\text { laboral }\end{array}$ & $\begin{array}{l}\text { Participación de } \\
\text { la productividad }\end{array}$ \\
\hline \multicolumn{8}{|l|}{ ECONOMÍAS EN DES ARROLLO } \\
\hline Total & 0.1274 & $\begin{array}{l}0.0031 \\
=\end{array}$ & $\begin{array}{c}0.0406 \\
=\end{array}$ & $\begin{array}{l}0.0837 \\
=\end{array}$ & & & \\
\hline \multicolumn{8}{|l|}{ Market services: } \\
\hline $\begin{array}{l}\text { Comercio al por mayor y al por menor; reparaciones; } \\
\text { hoteles y restaurantes; transportes. }\end{array}$ & $(0.020)$ & 0.0013 & 0.0203 & 0.0337 & 0.25 & 0.27 & 0.12 \\
\hline $\begin{array}{l}\text { Establecimientos financieros; bienes inmuebles } \\
\text { y servicios prestados a las empresas. }\end{array}$ & $(0.091)$ & 0.0003 & 0.0052 & 0.0053 & 0.16 & 0.04 & 0.56 \\
\hline No market services & $(0.016)$ & 0.0015 & 0.0150 & 0.0447 & 0.19 & 0.25 & 0.10 \\
\hline \multicolumn{8}{|l|}{ ECONOMÍAS DES ARROLLADAS } \\
\hline Total & 0.0334 & $\begin{array}{l}-0.0007 \\
=\end{array}$ & $\begin{array}{c}0.0188 \\
=\end{array}$ & $\begin{array}{l}0.0153 \\
=\end{array}$ & & & \\
\hline \multicolumn{8}{|l|}{ Market services: } \\
\hline $\begin{array}{l}\text { Comercio al por mayor y al por menor; reparaciones; } \\
\text { hoteles y restaurantes; transportes. }\end{array}$ & $(0.060)$ & -0.00070 & 0.00182 & 0.00312 & 0.22 & 0.28 & 0.12 \\
\hline $\begin{array}{l}\text { Establecimientos financieros; bienes inmuebles } \\
\text { y servicios prestados a las empresas. }\end{array}$ & $(0.250)$ & 0.00001 & 0.00169 & 0.00387 & 0.25 & 0.08 & 0.49 \\
\hline No market services & $(0.053)$ & -0.00002 & 0.01531 & 0.00828 & 0.28 & 0.39 & 0.11 \\
\hline
\end{tabular}

Fuente: Elaboración propia

La Tabla 5 muestra que el crecimiento de la productividad laboral en los subsectores del sector servicios depende del grado de desarrollo de las economías. En las economías desarrolladas, el crecimiento de la productividad depende principalmente del "efecto dinámico". En las economías en desarrollo, la productividad depende positivamente de los efectos "shift" y "within". Además, las estimaciones muestran que los mencionados efectos tienen una importancia mayor en las economías en desarrollo que en las desarrolladas. Por estas razones, los resultados podrían interpretarse en términos de hay una tendencia 
a que se reduzca el "efecto within" en las economías desarrolladas. Esto significa que el crecimiento de la productividad laboral en los subsectores del sector servicios tiende a reducirse cuando las economías se desarrollan.

La Tabla 5 también muestra que hay efectos dinámicos positivos en todos los subsectores del sector servicios tanto de las economías desarrolladas como de aquellas en desarrollo. Este hallazgo contrasta con los reportados en otros estudios, donde se muestra que únicamente los sub-sectores de finanzas, de comunicaciones, de transportes y de comercio contribuyen al crecimiento de la productividad laboral.

Las Tablas 4 y 5 también muestran que hay diferencias significativas entre las economías desarrolladas y en desarrollo. Sectorialmente, el "efecto within" siempre es mayor en las economías desarrolladas que en las economías en desarrollo. Además, las participaciones en el PIB y en el mercado laboral vinculadas al sector terciario también resultan mayores en las economías desarrolladas. Lo mismo ocurre con las contribuciones para el crecimiento de la productividad laboral del sector terciario y los subsectores del sector servicios. Así, la evidencia sugiere que el sector terciario sí contribuye, pero de manera parcializada, al incremento de la productividad global de las economías.

\subsection{Estimaciones econométricas}

En esta sección se analizan los resultados de las estimaciones de los grupos de regresiones usadas para evaluar los impactos de los servicios sobre el crecimiento de la productividad laboral. Las variables, los resultados de las estimaciones y los estadísticos de prueba se reportan en tablas. Particularmente, la Tabla 6 muestra las variables utilizadas, la Tabla 7 muestra las estimaciones y estadísticos del grupo de regresiones que evalúan los impactos usando los datos de la totalidad del sector terciario, la Tabla 8 muestra las estimaciones y estadísticos de las regresiones que evalúan los impactos usando los datos referidos a los servicios "Market services" y la Tabla 9 muestra las estimaciones y estadísticos de las regresiones que evalúan los impactos usando los datos referidos a los servicios "No market services". 
Tabla 6: Variables de panel de datos.

\begin{tabular}{|c|c|c|c|}
\hline VARIABLES & DEFINICIÓN & PERIODO & ECONOMÍAS \\
\hline gpcoun & Crecimiento de la productividad global de la economía & $2005-2009$ & 17 \\
\hline Inipms & Logaritmo de la productividad inicial de los "market services" & $2005-2009$ & 17 \\
\hline Inipnm & Logaritmo de la productividad inicial de los "no market services" & 2005-2009 & 17 \\
\hline Inips & Logaritmo de la productividad inicial del sector de los servicios & 2005-2009 & 17 \\
\hline Ingms & Logaritmo del crecimiento de la participación laboral de los "market services" & $2005-2009$ & 17 \\
\hline Ingnm & Logaritmo del crecimiento de la participación laboral de los "no market services" & 2005-2009 & 17 \\
\hline Ingss & Logaritmo del crecimiento de la participación laboral del sector de los servicios & 2005-2009 & 17 \\
\hline Inisms & Logaritmo de la participación laboral inicial de los "market services" & 2005-2009 & 17 \\
\hline Inis nm & Logaritmo de la participación laboral inicial de los "no market services" & 2005-2009 & 17 \\
\hline Iniss & Logaritmo de la participación laboral inicial del sector de los servicios & 2005-2009 & 17 \\
\hline Incapf & Logaritmo del Capital físico: formación bruta de capital fijo (\% del PIB) & $2005-2009$ & 17 \\
\hline Incaph & $\begin{array}{l}\text { Logaritmo del Capital humano: matrícula de educación primaria (\% bruto) }+ \text { matrícula de } \\
\text { educación secundaria (\% bruto) / índice del personal empleado mayor de } 15 \text { años } \\
\text { y la población total }(\%)\end{array}$ & 2005-2009 & 17 \\
\hline Indemo & $\begin{array}{l}\text { Logaritmo de la Composición demográfica: índice del personal empleado mayor de } 15 \text { años / } \\
\text { población total }(\%)\end{array}$ & 2005-2009 & 17 \\
\hline Intrao & $\begin{array}{l}\text { Logaritmo Apertura comercial: exportaciones de bienes y servicios (millones de US\$, } \\
\text { constante 2005) + importaciones de bienes y servicios (millones de US\$, constante } \\
\text { 2005) / PIB (millones de US\$, constante 2005) }\end{array}$ & 2005-2009 & 17 \\
\hline develp & $\begin{array}{l}\text { Variable dummy del grado de desarrollo } \\
\text { (Economía en desarrollo }=1 \text {, economía desarrollada }=0 \text { ) }\end{array}$ & 2005-2009 & 17 \\
\hline
\end{tabular}

Fuente: Elaboración propia

Nota: Las economías de Brasil y Malasia fueron exclui das por la falta de disponibilidad de datos

Tabla 7: Cambio estructural y crecimiento de la productividad del sector terciario (2005-2009) (Datos en panel con efectos aleatorios, forma funcional lin-log)

\begin{tabular}{|c|c|c|c|c|c|c|}
\hline \multirow{2}{*}{$\begin{array}{l}\text { Variable dependiente: gpcoun } \\
\text { Variables independientes: } \\
\end{array}$} & \multicolumn{2}{|c|}{ Economías en desarrollo (10) } & \multicolumn{2}{|c|}{ Economías desarrolladas (7) } & \multicolumn{2}{|c|}{ Total de economías (17) } \\
\hline & Estimación & Semi-elasticidad & Estimación & Semi-elasticidad & Estimación & Semi-elasticidad \\
\hline Iniss & $\begin{array}{c}0.1301 \\
(0.46)\end{array}$ & 3.79 & $\begin{array}{c}0.6607 \\
(1.08)\end{array}$ & 19.25 & $\begin{array}{c}0.2358 \\
(1.10)\end{array}$ & 6.87 \\
\hline Incaph & $\begin{array}{l}-1.1127 \\
(1.58)\end{array}$ & -32.42 & $\begin{array}{l}-1.1772 \\
(1.36)\end{array}$ & -34.29 & $\begin{array}{l}-0.6188 \\
(1.53)\end{array}$ & -18.03 \\
\hline Indemo & $\begin{array}{l}-1.491 * * \\
(2.07)\end{array}$ & $-43.43 * *$ & $\begin{array}{l}-1.2227 \\
(1.19)\end{array}$ & -35.62 & $\begin{array}{l}-0.8878 * \\
(1.90)\end{array}$ & $-25.86 *$ \\
\hline Intrao & $\begin{array}{l}-0.0102 \\
(0.21)\end{array}$ & -0.30 & $\begin{array}{c}0.1121 \\
(0.99)\end{array}$ & 3.27 & $\begin{array}{l}-0.038 \\
(1.22)\end{array}$ & -1.11 \\
\hline
\end{tabular}

Fuente: Elaboración propia

Nota: La variable dependiente es el crecimiento de la productividad global de la economía (gpcoun). Las constantes fueron estimadas, pero no se muestran en las regresiones. Los errores estándar se indican entre paréntesis. Uno, dos y tres asteriscos indican, respectivamente niveles de significancia del 10,5 y 1 por ciento 
Tabla 8: Cambio estructural y crecimiento de la productividad de los servicios "market services" (2005-2009) (Datos en panel con efectos aleatorios, forma funcional lin-log)

\begin{tabular}{|c|c|c|c|c|c|c|}
\hline \multirow{2}{*}{$\begin{array}{l}\text { Variable dependiente: gpcoun } \\
\text { Variables independientes: }\end{array}$} & \multicolumn{2}{|c|}{ Economías en desarrollo (10) } & \multicolumn{2}{|c|}{ Economías desarrolladas (7) } & \multicolumn{2}{|c|}{ Total de economías (17) } \\
\hline & Estimación & Semi-elasticidad & Estimación & Semi-elasticidad & Estimación & Semi-elasticidad \\
\hline Ingms & $\begin{array}{l}-0.0049 \\
(0.37)\end{array}$ & -0.09 & $\begin{array}{c}0.0164 \\
(0.23)\end{array}$ & 1.85 & $\begin{array}{l}-0.0123 \\
(0.87)\end{array}$ & -0.36 \\
\hline Inipms & $\begin{array}{l}-0.0211 \\
(0.74)\end{array}$ & -0.61 & $\begin{array}{c}0.4417 \\
(0.65)\end{array}$ & 12.87 & $\begin{array}{l}-0.0116 \\
(0.51)\end{array}$ & -0.34 \\
\hline Inisms & $\begin{array}{c}0.1795 \\
(0.87)\end{array}$ & 5.23 & $\begin{array}{c}0.0867 \\
(0.13)\end{array}$ & 2.53 & $\begin{array}{c}0.0994 \\
(0.94)\end{array}$ & 2.90 \\
\hline Incapf & $\begin{array}{c}0.3094 \text { *** } \\
(3.00)\end{array}$ & $9.01 * * *$ & $\begin{array}{c}0.4178 \\
(0.79)\end{array}$ & 12.17 & $\begin{array}{c}0.2473 * * \\
(2.27)\end{array}$ & $7.20 * *$ \\
\hline Incaph & $\begin{array}{c}-1.8574 * * \\
(2.64)\end{array}$ & $-54.11 * *$ & $\begin{array}{l}-1.0737 \\
(0.64)\end{array}$ & -31.28 & $\begin{array}{l}-0.6803 \\
(1.37)\end{array}$ & -19.82 \\
\hline Indemo & $\begin{array}{c}-2.3314 \text { *** } \\
(3.40)\end{array}$ & $-67.91 * * *$ & $\begin{array}{l}-0.5424 \\
(0.41)\end{array}$ & -15.80 & $\begin{array}{c}-0.9959 * * \\
(2.09)\end{array}$ & $-29.01 * *$ \\
\hline Intrao & $\begin{array}{c}0.0061 \\
(0.15)\end{array}$ & 0.18 & $\begin{array}{c}0.2892 \\
(0.76)\end{array}$ & 8.43 & $\begin{array}{l}-0.0349 \\
(1.14)\end{array}$ & -1.02 \\
\hline & $\begin{array}{r}\text { Observaciones: } 43 \\
\text { Wald: } 21.54 * * *\end{array}$ & $\begin{array}{c}\text { Jarque-Bera } 1.38 \\
\text { Hausman } 8.02\end{array}$ & $\begin{array}{c}\text { Observaciones: } 17 \\
\text { b Wald } 1.05\end{array}$ & $\begin{array}{c}\text { Jarque-Bera } 4.06 \\
\text { Hausman } 3.22\end{array}$ & $\begin{array}{r}\text { Observaciones: } 60 \\
\text { Wald: } 13.21 * * *\end{array}$ & $\begin{array}{l}\text { Jarque-Bera } 1.33 \\
\text { Hausman } 11.05\end{array}$ \\
\hline
\end{tabular}

Fuente: Elaboración propia

Nota: La variable dependiente es el crecimiento de la productividad global de la economía (gpcoun). Las constantes fueron estimadas, pero no se muestran en las regresiones. Los errores estándar se indican entre paréntesis. Uno, dos y tres asteriscos indican, respectivamente niveles de significancia del 10, 5 y 1 por ciento

Tabla 9: Cambio estructural y crecimiento de la productividad de los servicios "market services" (2005-2009) (Datos en panel con efectos aleatorios, forma funcional lin-log)

\begin{tabular}{|c|c|c|c|c|c|c|}
\hline \multirow{2}{*}{$\begin{array}{l}\text { Variable dependiente: gpcoun } \\
\text { Variables independientes: }\end{array}$} & \multicolumn{2}{|c|}{ Economías en des arrollo (10) } & \multicolumn{2}{|c|}{ Economías desarrolladas (7) } & \multicolumn{2}{|c|}{ Total de economías (17) } \\
\hline & Estimación & Semi-elasticidad & Estimación & Semi-elasticidad & Estimación & Semi-elasticidad \\
\hline Ingnm & $\begin{array}{c}-0.0297 * * \\
(2.39)\end{array}$ & $-0.52 * *$ & $\begin{array}{c}0.0071 \\
(0.35)\end{array}$ & 0.80 & $\begin{array}{c}-0.0264 * * \\
(2.54)\end{array}$ & $-0.76 * *$ \\
\hline Inipnm & $\begin{array}{l}-0.0348 \\
(0.34)\end{array}$ & -1.01 & $\begin{array}{l}0.3897 * \\
(1.77)\end{array}$ & $11.35 *$ & $\begin{array}{c}0.0032 \\
(0.07)\end{array}$ & 0.09 \\
\hline Inisnm & $\begin{array}{l}-0.0194 \\
(0.07)\end{array}$ & -0.57 & $\begin{array}{c}0.1161 \\
(0.71)\end{array}$ & 3.38 & $\begin{array}{l}-0.0590 \\
(0.47)\end{array}$ & -1.72 \\
\hline Incapf & $\begin{array}{c}0.1649 \\
(1.45)\end{array}$ & 4.80 & $\begin{array}{c}0.3683 * * \\
(2.15)\end{array}$ & $10.72 * *$ & $\begin{array}{c}0.0704 \\
(0.90)\end{array}$ & 2.05 \\
\hline Incaph & $\begin{array}{l}-1.6051 \\
(1.61)\end{array}$ & -46.76 & $\begin{array}{l}-0.6367 \\
(1.05)\end{array}$ & -18.55 & $\begin{array}{l}-0.4262 \\
(0.91)\end{array}$ & -12.42 \\
\hline Indemo & $\begin{array}{l}-1.9182 * \\
(1.91)\end{array}$ & $-55.88 *$ & $\begin{array}{l}-0.1612 \\
(0.27)\end{array}$ & -4.70 & $\begin{array}{l}-0.4524 \\
(0.99)\end{array}$ & -13.18 \\
\hline Intrao & $\begin{array}{c}0.0467 \\
(0.82)\end{array}$ & 1.36 & $\begin{array}{c}0.0969 * \\
(1.75)\end{array}$ & $2.82 *$ & $\begin{array}{l}-0.0085 \\
(0.30)\end{array}$ & -0.25 \\
\hline & Observaciones: 33 & Jarque-Bera 1.95 & Observaciones: 30 & Jarque-Bera 4.49 & Observaciones: 63 & Jarque-Bera $7.47 * *$ \\
\hline & Wald: $19.82 * * *$ & Hausman $13.96 *$ & Wald: $9.75 * * *$ & Hausman 3.24 & Wald: $7.47 * * *$ & ${ }^{\mathrm{c}}$ Hausman $56.45 * * *$ \\
\hline
\end{tabular}

Fuente: Elaboración propia

Nota: La variable dependiente es el crecimiento de la productividad global de la economía (gpcoun). Las constantes fueron estimadas, pero no se muestran en las regresiones. Los errores estándar se indican entre paréntesis. Uno, dos y tres asteriscos indican, respectivamente niveles de significancia del 10, 5 y 1 por ciento 
Las Tablas 7, 8 y 9 sugieren que el crecimiento de la participación de los servicios en los mercados de trabajo tiene pocas relaciones con el crecimiento de la productividad de la economía. La mayoría de los coeficientes estimados son no significativos. Las excepciones refieren a los coeficientes estimados para el grupo de regresiones de los servicios "No market services" (véase la Tabla 9). Estas últimas regresiones muestran relaciones negativas entre la participación de los servicios en el mercado de trabajo y la productividad en las economías en desarrollo. La evidencia, por tanto, muestra que no todos los sub-sectores del sector servicios contribuyen de la misma manera en el proceso de crecimiento económico. Por tanto, la hipótesis de que los servicios tienen una contribución negativa en la productividad tiene una validez limitada.

Las Tablas 7, 8 y 9 también sugieren que las condiciones iniciales de la participación de los servicios en los mercados de trabajo y de la productividad tienen pocas relaciones con el crecimiento de la productividad de la economía. La mayoría de los coeficientes estimados son no significativos. Las excepciones nuevamente refieren a los coeficientes estimados para el grupo de regresiones de los servicios "No market services" (véase la Tabla 9). Estas últimas regresiones muestran relaciones positivas entre la productividad inicial y la productividad para las economías desarrolladas. La evidencia, por tanto, no valida la hipótesis de convergencia. Además, la mayoría de las estimaciones muestran que la inversión en capital físico es importante para incrementar la productividad global.

Las tablas también sugieren que hay relaciones diferenciadas entre los capitales y el crecimiento de la productividad en las economías desarrolladas y en desarrollo. Las estimaciones para las economías en desarrollo muestran que hay correlaciones positivas y significativas entre el capital físico y la productividad para los servicios "Market services". Sin embargo, también muestran que hay correlaciones negativas y significativas entre el capital humano y la productividad para los servicios "Market services". Además, las estimaciones muestran que hay correlaciones negativas entre la edad de los trabajadores y la productividad.

Las estimaciones también muestran algunos resultados complementarios para las economías desarrolladas. Entre estos se incluyen los siguientes: 1) En las estimaciones para los servicios "No market services" hay correlaciones positivas 
y significativas entre el capital físico y la apertura comercial con la productividad, y 2) no hay correlaciones entre el capital humano y la edad de los trabajadores con la productividad.

\section{Conclusiones y discusión}

Esta investigación ha mostrado que la terciarización ha sido importante en las economías analizadas. Particularmente, el análisis de estadística descriptiva ha mostrado que la participación de los servicios y la productividad laboral han sido mayores en las economías desarrolladas que en las economías en desarrollo. También ha mostrado que la productividad en las economías desarrolladas ha sido 3.24 veces más alta en las economías desarrolladas que en sus contrapartes en desarrollo. Además, la evidencia sugiere que ha habido un proceso de terciarización para todas las economías analizadas. Incluso, para las economías en desarrollo, la participación de los servicios en los mercados de trabajo ha sido creciente durante el periodo estudiado. Por tanto, la evidencia sugiere que todas economías experimentaron un proceso de reubicación laboral hacia el sector terciario.

El análisis shift-share ha mostrado que el crecimiento de la productividad laboral a nivel sectorial ha dependido "efecto within" tanto para las economías desarrolladas como para sus contrapartes en desarrollo. Sin embargo, para el sector terciario, el análisis también muestra que el "efecto shift" ha contribuido marginalmente a promover su productividad. El análisis también sugiere que el crecimiento de la productividad laboral en los subsectores se reduce cuando hay un mayor desarrollo de las economías. Además, el análisis sugiere que el sector terciario contribuye parcialmente a incrementar la productividad global y a promover el desarrollo de las economías vía los servicios "No market services". ${ }^{19}$ De hecho, la evidencia muestra que hay efectos dinámicos positivos en todos los subsectores del sector servicios.

Elanálisis econométrico permite evaluar algunas hipótesis sobre los determinantes del crecimiento de la productividad global. Particularmente, el análisis muestra que la participación y condiciones iniciales de los servicios en los mercados de

19 Adviértase que esta conclusión difiere de aquella obtenida por Inklaar y Timmer (2014). Ellos hallan que los servicios 'Market services' han sido contribuyentes al crecimiento económico de varias economías desarrolladas. 
trabajo tienen pocas relaciones con la productividad. Por tanto, la evidencia muestra que la hipótesis de que los servicios tienen una contribución negativa en la productividad tiene una validez limitada. El análisis también muestra que las condiciones iniciales de la productividad tienen pocas relaciones con la productividad. Por tanto, la evidencia no valida la hipótesis de convergencia.

El análisis también sugiere que hay relaciones diferenciadas entre los determinantes del crecimiento de la productividad en las economías desarrolladas y en desarrollo. Particularmente, las estimaciones para las economías en desarrollo muestran que hay correlaciones positivas y significativas entre el capital físico y la productividad para los servicios "Market services". Las estimaciones para las economías desarrolladas muestran que hay correlaciones positivas y significativas entre el capital físico y la apertura comercial con la productividad para los servicios "No market services". Además, la evidencia muestra que un determinante importante de la productividad global es la inversión en capital físico. Finalmente, debe destacarse que el análisis sugiere que no todos los subsectores del sector servicios contribuyen de la misma manera en el proceso de crecimiento económico. ${ }^{20}$ Aparentemente, los servicios "No market services" tienen características intrínsecas que no se reflejan en los indicadores de productividad. ${ }^{21}$

Metodológicamente, debe señalarse que esta investigación constituye una aproximación al análisis del papel de los servicios en las economías desarrolladas y en desarrollo. Por tanto, no sobra señalar que los resultados deben considerarse como preliminares. La primera razón que justifica esta consideración es porque existen problemas teóricos que limitan la comparabilidad entre las economías con diferente grado de desarrollo. De hecho, hay quienes argumentan que las economías en desarrollo tienen diferencias de productividad entre sus sectores e industrias que no son comparables con sus contrapartes de las economías desarrolladas (McMillan y Rodrik, 2011).

Una segunda razón que justifica que los resultados sean preliminares se vincula a la suposición implícita de que el sector informal no tiene importancia en las

20 Adviértase que Jorgenson y Timmer (2011), a diferencia nuestra, hallan que los servicios, de manera general, tienen un papel importante en el crecimiento de la productividad.

21 Estas características incluyen la intangibilidad, la temporalidad, la co-producción y la simultaneidad. Véase los estudios de Djellal y Gallouj (2008) y McMillan y Rodrik (2011). 
economías. Esta suposición, si bien restrictiva, se asume debido a la falta de los indicadores pertinentes. En este contexto, no sobra señalar que Timmer y de Vries (2009) enfatizan que las actividades informales pueden introducir sesgos en las estimaciones. Por esta razón, debe reconocerse que las actividades informales podrían explicar las diferencias entre las economías desarrolladas y en desarrollo. Además, los sistemas fiscales ineficientes y los problemas de regulación propios de las economías en desarrollo podrían agudizar las mencionadas diferencias (Schneider, et al., 2010).

Una tercera razón que justificaría que los resultados fueran considerados como preliminares, se asocia a que ha habido una tendencia creciente en el intercambio comercial a nivel global. ${ }^{22}$ Según McMillan y Rodrik (2011), la apertura comercial no es, por sí sola, una explicación al crecimiento de la productividad. La apertura comercial depende de la localización de las plantas de producción, de las elecciones realizadas por los responsables de las políticas públicas y de las estrategias internas de crecimiento. Todos estos factores dependen de prácticas de gobernanza que funcionen adecuadamente. Sin embargo, en las economías en desarrollo dichas prácticas suelen ser imperfectas. Esta consideración podría explicar porque la apertura comercial es un determinante de la productividad global solo en las economías desarrolladas.

Finalmente, no sobra enfatizar que los resultados de esta investigación muestran la pertinencia de realizar mayor investigación sobre el sector terciario. Se necesitan más estudios para validar y generalizar los hallazgos reportados y esta no será una tarea sencilla. La realización de nuevos análisis del tipo shiftshare dependerá de que haya mejoras en las contabilidades nacionales a nivel sectorial. Nuevos análisis econométricos, por su parte, muy probablemente requerirán el uso de técnicas para paneles dinámicos y la inclusión de controles referidos a las distintas dimensiones de la gobernanza. La justificación de dichos estudios podría plantearse en términos de la mejora de las políticas públicas y de la mejora de la planeación macroeconómica. Por estas razones, consideramos que esta investigación podría tomarse como un referente para promover nuevos estudios sobre el sector terciario y el desarrollo económico.

22 El periodo de los 90's al 2005 ha sido uno en el que la globalización ha impactado de mayor manera a las economías en desarrollo. Los aranceles han bajado y los flujos de inversión extranjera se han incrementado (McMillan y Rodrik, 2011). 


\section{APÉNDICE}

Clasificación Industrial Internacional Uniforme de todas las actividades económicas, Rev. 3.1. ( Fuente: División de estadísticas de las Naciones Unidas).

\section{A Agricultura, ganadería, caza y silvicultura}

01 - Agricultura, ganadería, caza y actividades de servicios conexas.

02 - Silvicultura, extracción de madera y actividades de servicios conexas.

B Pesca

05 - Pesca, acuicultura y actividades de servicios relacionadas con la pesca.

C Explotación de minas y canteras

10 - Extracción de carbón y lignito; extracción de turba.

11 - Extracción y servicios de petróleo crudo y gas natural; excepto las actividades de prospección.

12 - Extracción de minerales de uranio y torio.

13 - Extracción de minerales metalíferos.

14 - Explotación de otras minas y canteras.

D Industrias manufactureras

15 - Elaboración de productos alimenticios y bebidas.

16 - Elaboración de productos de tabaco.

17 - Fabricación de productos textiles.

18 - Fabricación de prendas de vestir; adobo y teñido de pieles.

19 - Curtido y adobo de cueros; artículos de talabartería y guarnicionería, y calzado.

20 - Producción de madera y fabricación de productos de madera y corcho, excepto muebles.

21 - Fabricación de papel y de productos de papel.

22 - Actividades de edición e impresión y de reproducción de grabaciones.

23 - Fabricación de coque, productos de la refinación del petróleo y combustible nuclear. 
24 - Fabricación de sustancias y productos químicos.

25 - Fabricación de productos de caucho y plástico.

26 - Fabricación de otros productos minerales no metálicos.

27 - Fabricación de metales comunes.

28 - Fabricación de productos elaborados de metal, excepto maquinaria y equipo.

29 - Fabricación de maquinaria y equipo n.c.p (no clasificado previamente).

30 - Fabricación de maquinaria de oficina, contabilidad e informática.

31 - Fabricación de maquinaria y aparatos eléctricos n.c.p.

32 - Fabricación de equipo y aparatos de radio, televisión y comunicaciones.

33 - Fabricación de instrumentos médicos, ópticos y de precisión y fabricación de relojes.

34 - Fabricación de vehículos automotores, remolques y

semirremolques.

35 - Fabricación de otros tipos de equipo de transporte.

36 - Fabricación de muebles; industrias manufactureras n.c.p.

37 - Reciclado.

E Suministro de electricidad, gas y agua

40 - Suministro de electricidad, gas, vapor y agua caliente.

41 - Captación, depuración y distribución de agua.

F Construcción

45 - Construcción.

G Comercio al por mayor y al por menor; reparación de vehículos, motocicletas, y enseres domésticos.

50 - Venta, mantenimiento y reparación de vehículos automotores y motocicletas.

51 - Comercio al por mayor y en comisión, excepto el comercio de vehículos.

52 - Comercio al por menor, excepto el comercio de vehículos.

H Hoteles y restaurantes

55 - Hoteles y restaurantes.

I Transporte, almacenamiento y comunicaciones 
60 - Transporte por vía terrestre; transporte por tuberías.

61 - Transporte por vía acuática.

62 - Transporte por vía aérea.

63 - Actividades de transporte complementarias y auxiliares;

actividades de agencias de viajes.

64 - Correo y telecomunicaciones.

J Intermediación financiera

65 - Intermediación financiera, excepto la financiación de planes de seguros y de pensiones.

66 - Financiación de planes de seguros y de pensiones, excepto los planes de seguridad social.

67 - Actividades auxiliares de la intermediación financiera.

K Actividades inmobiliarias, empresariales y de alquiler

70 - Actividades inmobiliarias.

71 - Alquiler de maquinaria y equipo sin operarios y de efectos personales y enseres domésticos.

72 - Informática y actividades conexas.

73 - Investigación y desarrollo.

74 - Otras actividades empresariales.

L Administración pública y defensa; planes de seguridad social de afiliación obligatoria

75 - Administración pública y defensa; planes de seguridad social de afiliación obligatoria.

M Enseñanza

80 - Enseñanza.

N Servicios sociales y de salud

85 - Servicios sociales y de salud.

0 Otras actividades de servicios comunitarios, sociales y personales

90 - Eliminación de desperdicios y aguas residuales, saneamiento y actividades similares.

91 - Actividades de asociaciones n.c.p.

92 - Actividades de esparcimiento y actividades culturales y deportivas. 
93 - Otras actividades de servicios.

P Actividades de hogares privados como empleadores

95 - Actividades de hogares privados como empleadores de personal doméstico.

96 - Actividades no diferenciadas de hogares privados como productores de bienes para uso propio.

97 - Actividades no diferenciadas de hogares privados como productores de servicios (uso propio).

Q Organizaciones y órganos extraterritoriales

99 - Organizaciones y órganos extraterritoriales. 


\section{Referencias bibliográficas}

- Barro, R. y Sala-i-Martin, X. (2004). Economic Growth. Cambridge: MIT Press.

- Baumol, W. (1967). "Macroeconomics of unbalanced growth: The anatomy of urban crisis". American Economic Review, 57 (3), 416-426.

- Baumol, W. (2002). "Services as Leaders and the Leader of the Services". En J. Gadrey y F. Gallouj (ed.), Productivity, Innovation and Knowledge in Services. Cheltenham: Edward Elgar.

- Bhagwati, J. (1984). "Why are services cheaper in the poor countries?" The Economic Journal, 94(374), 279-86.

- Cavalcanti-Ferreira, P. y Rossi, J. L. (2003) "New evidence from Brazil on trade, liberalization and productivity growth". International Economic Review, 44(4), 1383-1405.

- Chung, W. y Denison, E. (1976). How Japanese Economy Grew so Fast: The Sources of Post-war Expansion. Washington, D.C.: Brookings Institution.

- Clark, C. (1940). The Conditions of Economic Progress. London: Macmillan.

- Dabla-Norris, E., Alun, T., Garcia-Verdu, R. y Yingyuan, C. (2013). "Benchmarking structural transformation across the world". Working Paper No. 13/176. International Monetary Fund. Washington, DC.

- Dabla-Norris, E., Giang, H., Kochhar, K., Kyobe, A. y Tchaidze, R. (2014). "Anchoring growth: The importance of productivity-enhancing reforms in emerging market and developing economies". Journal of International Commerce, Economics and Policy, 5 (2), 1-29.

- Denison, E. (1967). Why Growth Rates Differ: Post-war Experience in Nine Western Countries. Washington, D.C.: Brookings Institution.

- Djellal, F. y Gallouj, F. (2008). Measuring and Improving Productivity in Services. Cheltenham: Edward Elgar Publishing Limited.

- Esclava, M., Haltiwanger, J., Kugler, A. D. y Kugler, M.(2009). "Trade reforms and market selection: Evidence from manufacturing plants in Colombia". NBER Working Paper No 14935.

- Faberger, J. (2000). "Technological progress, structural change and productivity growth: a comparative study". Structural Change and Economic Dynamics, 11(4), 393-411. 
- Fernandes, A. M. (2007). "Trade policy, trade volumes and plant-level productivity in Colombian manufacturing industries". Journal of International Economics, 71(1), 52-71.

- Feinstein, C. (1999). "Structural change in the developed countries during the twentieth century". Oxford Review of Economic Policy, 15(4), 35-55.

- Fisher, A. (1935). The Clash of Progress and Security. New York: Augustus M. Kelley.

- Fisher, A. (1939). "Production, primary, secondary and tertiary". Economic Record, 15(1), 24-38.

- Fourastié, J. (1949). "Le grand espoir du XXeme siècle". En Progrés Technique, Progress Économique, Progres Social. Paris : Presses Universitaires de France.

- Fuchs, V. (1968). The Service Economy. New York : Columbia University Press.

- Gemmell, N. (1982). "Economic development and structural change: the role of the service sector". Journal of Development Economics, 19(1), 37-66.

- Gershuny, J. y Miles, I. (1983). The New Service Economy. London: Frances Pinter.

- Howe, W. (1986). "The business services industry sets pace in employment growth". Monthly Labor Review, 109(4), 29-36.

- Hsieh, C.T. y Klenow, P. (2009). "Misallocation and manufacturing TFP in India and China". Quarterly Journal of Economics, 124(4), 1403-1448.

- Inklaar, R. y Timmer, M. (2014). "The relative price of services". Review of Income and Wealth, 60 (4), 727-746.

- Johnston, J. (1975). "A macro-model of inflation". Economic Journal, 85 (338), 288-308.

- Jorgenson, D. y Marcel, T. (2011). "Structural Change in Advanced Nations: A new set of stylized facts”. Scandinavian Journal of Economics, 113(1), $1-29$.

- Knudsen, D. (2000). "Shift-share analysis: further examination of models for the description of economic change". Socio-Planning Sciences, 34(3), 177198.

- Kutscher, R. y Personick, V. (1986). "Deindustrialization and shift to services". Monthly Labor Review, 109(1), 3-13. 
- Kuznets, S. (1957). "Quantitative aspects of the economic growth of nations: Industrial distribution of national product and labour force". Economic Development and Cultural Change, 5(4), 1-111.

- Kuznets, S (1966). Modern Economic Growth. Connecticut: Yale University Press.

- Maddison, A. (1980). "Economic Growth and Structural Change in Advanced Countries". En I. Levenson y J. Wheeler (ed.), Western Economies in Transition: Structural Change and Adjustment Policies Industrial Countries. Boulder, Westview Press.

- Maroto, A. y Cuadrado, J. (2007). "Productivity and tertiarization in industrialized countries: A comparative analysis". Efficiency Working Series, 15. University of Oviedo.

- Maroto, A. y Cuadrado, J. (2009). "Is growth of services an obstacle to productivity growth?". Structural Change and Economic Dynamics, 20(4), 254-265.

- Maroto, A. y Cuadrado, Juan. (2011). "Analyzing the role of service sector on productivity growth across European regions". Institute of Social and Economic Analysis, 4. University of Alcalá.

- McMillan, M. y Rodrik, D. (2011). "Globalization, structural change and productivity growth". Working Paper 17143. National Bureau of Economic Research. Cambridge.

- Mazumdar, D. (1976). "The urban informal sector". World Development, 4(8), 655-679.

- Nurkse, R. (1955). Problemas de Formación de Capital en los Países Insuficientemente Desarrollados. Mexico D.F., Fondo de Cultura Económica.

- O'Mahony, M. y Ark, B. Van (2003). "EU productivity and competitiveness: An industry perspective. Can Europe resume the catching-up process?" Enterprise publications. Brussels: European Commission.

- Pandit, K. (1989). "Service labor allocation during development: longitudinal perspectives on cross-sectional patterns". The Annals of Regional Science, 24(1), 29-41.

- Paus, E., Reinhardt, N. y Robinson, M. D. (2003). "Trade liberalization and productivity growth in Latin American manufacturing, 1970-98". Journal of Policy Reform, 6(1), 1-15. 
- Pavcnik, N. (2000). "Trade liberalization, exit, and productivity improvements: Evidence from Chilean plants”. NBER Working Paper No. 7852.

- Peneder, M., Kaniovski, S. y Dachs, B. (2003). "What follows tertiarization? Structural change and the role of knowledge-based services". The Service Industries Journal, 23(2), 47-66.

- Ramos, J. (1970). Labor and Development in Latin America. New York: Columbia University Press.

- Salter, W. (1960). Productivity and Technical Change. Cambridge: Cambridge University Press.

- Schneider, F. Buehn, A. y Montenegro, C. (2010). "Shadow economies all over the world: New estimates for 162 countries from 1999 to 2007". Policy Research Working Paper 5356: The World Bank, Development Research Group Poverty and Inequality Team \& Europe and Central Asia Region Human Development Economics Unit.

- Squire, L. (1979). "Labor force, employment and labor markets in the course of economic development". World Bank Staff Working Paper 336. Washington: World Bank.

- Stigler, G. (1956). "Trends in employment in the service industries". National Bureau of Economic Research General Series, 59. Princeton: Princeton University Press.

- Stiroh, K. (2002). "Information technology and the US productivity revival: What do the industry data say?" American Economic Review, 92(5), 15591576.

- Temple, J. y Woessmann, L. (2006). "Dualism and cross-country growth regressions". Journal of Economic Growth, 11(3), 187-228.

- Timmer, M. y Szirmai, A. (2000). "Productivity growth in Asian manufacturing: The structural bonus hypothesis examined". Structural Change and Economic Dynamics, 11(4), 371-392.

- Timmer, M. y de Vries, G. (2009). "Structural change and growth accelerations in Asia and Latin America: A new sectoral data set". Journal of Historical Economics and Econometric History, 3(2), 165-190.

- Wolszczak-Derlacz, J. (2014). "The impact of domestic and foreign competition on sectoral growth: A cross-country analysis". Bulletin of Economic Research, 66 (S1), S110-S131. 
- Wooldridge, J.M. (2010). Econometric Analysis of Cross Section and Panel Data. Segunda Edición. Cambridge: The MIT Press.

- World Bank. (2007). Global Economic Prospects 2007: Managing the Next Wave of Globalization. Washington DC: International Bank for Reconstruction and Development. 